Short report

\title{
The impact of computed tomography on the treatment of chronic subdural haematoma
}

\author{
AH MOUSSA, N JOSHY \\ From the Department of Neurological Surgery, Salford Royal Hospital, Salford, UK
}

SUMMARY Twenty four consecutive patients with chronic subdural haematoma were treated by burr hole evacuation. Pre-operative and post-operative CT scans were performed and all patients developed a re-collection of fluid within the first week. This did not adversely influence clinical improvement and resolved spontaneously.

Chronic subdural haematoma often occurs as a consequence of minor trauma and is usually due to tearing of one of the veins that bridge the subdural space. ${ }^{1,2}$ The cells of the inner surface of the dura then proliferate and the blood clot becomes encapsulated within a subdural membrane. Secondary events will then determine the clinical outcome; the thin-walled sinusoidal channels of the outer membrane have loose cell junctions and are thus liable to further bleeding, ${ }^{3,4}$ but the membrane also functions as an absorptive surface which tends to reduce haematoma size. ${ }^{2}$ Indeed, spontaneous absorption of subdural haematoma has been demonstrated clinically. ${ }^{5,6}$ The absorptive ability of the membrane may be directly related to its surface area. However, recurrent minor trauma may provoke rebleeding and the outcome in any particular patient will depend on the balance between absorption and expansion of the haematoma.

A wide variety of therapeutic approaches to the treatment of chronic subdural haematomas have been advocated. Craniotomy and membranectomy were considered necessary by Robinson, ' but this view was challenged by Svien and Gelety who, in a comparative study of membranectomy versus simple burr hole evacuation, demonstrated that the latter procedure was superior to craniotomy. ${ }^{8}$ Tabaddor and Shulman ${ }^{9}$ compared these two surgical treatments with twist-drill craniotomy and closed system drainage in a total of 71 patients and showed that the latter method produced the better results. Non-surgical treatment with bed rest and mannitol therapy was advocated by Bender and Christoff. ${ }^{10}$

Address for reprint requests: Dr Abdel Hai Moussa, FRCS, Department of Neurosurgery, Faculty of Medicine, Ain-Shams University, Cairo, Egypt

Received 5 May 1982 and in revised form 20 June 1982. Accepted 9 August 1982.
Thus controversy still exists about the management of chronic subdural haematoma, and many of the studies were performed in the era prior to CT scanning which has revolutionised the diagnosis of haematoma. The present study was designed to monitor the events following the evacuation of the haematoma by burr hole.

\section{Patients and methods}

Twenty-four consecutive patients form the basis of this study. They were admitted under the care of one neurological surgeon from 1976, when CT scanning facilities were installed, to 1980 . All had CT scan appearances consistent with chronic subdural haematoma and all proved to have a unilateral lesion. Children under the age of 13 years and patients in whom an infectious disorder (subdural empyema) was suspected were excluded. Also excluded were all patients in whom it was considered possible that the subdural haematoma might be associated with a haemorrhagic tendency.

Of the 24 patients, 17 were male and seven female. The age range was 13 to 76 years. Seventeen of them were more than 50 years of age. A direct head injury was identified in 14 patients and of the remaining ten, two had long-standing epilepsy and two were alcoholic. A further two patients abused alcohol in the context of a chronic psychiatric disturbance. Another patient had taken several overdoses, but no causative or precipitating factor could be identified in the three remaining patients. The symptoms-to-operation period in these three patients was only one week, but they were included in this study in view of their clinical presentation, CT scan picture and operative findings. The trauma-to-operation or symptoms-to-operation interval in the other twenty-one patients varied from six weeks to one year. Seven patients were in a coma 
(no verbal response and only flexion response in the limbs to painful stimuli) by the time of operation. Five of these had a fixed, dilated pupil. Hemiparesis was the common abnormal neurological sign and was the main feature in nine patients, together with either dysphasia or intellectual change or both. Of the remaining eight patients, six presented with features of raised intracranial pressure and two had intellectual change only. In 16 patients two burr holes were made over the haematoma. In eight patients only one was made. Complete evacuation was aided by saline irrigation. The haematoma depressed the cortex by $2 \mathrm{~cm}$ or more in 14 patients and by only $1 \mathrm{~cm}$ in the rest of the patients. Full re-expansion of the brain at the time of operation was achieved only in seven patients in whom isotonic saline had been injected by lumbar puncture when the dura was still open. This was done early in the series and the patients were amongst those who had two burr holes. None of the patients was subjected to further drainage. Early mobilisation was encouraged after operation and the hospitalisation period ranged from 3 to 13 days, with an average of seven days. Four CT scans were performed on each patient, the first prior to surgery, the second within one week after surgery, the third after about 4 weeks after operation and the fourth after an interval of 8 to 12 weeks.

\section{Results}

The initial CT scan confirmed the presence of chronic subdural haematoma in every patient (fig (a)). When the study was repeated within the first post-operative week, residual collection with some midline shift was identified in every case (fig (b)), including those in whom full re-expansion of the brain was achieved operatively. Despite these appearances, all the patients were better than prior to surgery. By the fourth post-operative week, the CT scan appearance had considerably improved, but still showed some subdural collection in all cases, associated with midline shift in some patients (fig (c)). The residual collection invariably had a lower density than that of the brain. At 8 to 12 weeks, there was resolution of the subdural collection and full expansion of the brain in every case (fig (c)). However, the final CT scan appearances suggested a minor degree of cortical atrophy in many of the patients. This may have been present before the development of chronic subdural haematoma and thus its significance is uncertain.

Twenty-three patients made a complete recovery and returned to their normal state of health within 8 weeks of surgery. One patient, a 71-year-old man who was unconscious with fixed and dilated pupils at time of surgery, made a good recovery but died two weeks after discharge of fulminating bronchopneumonia.

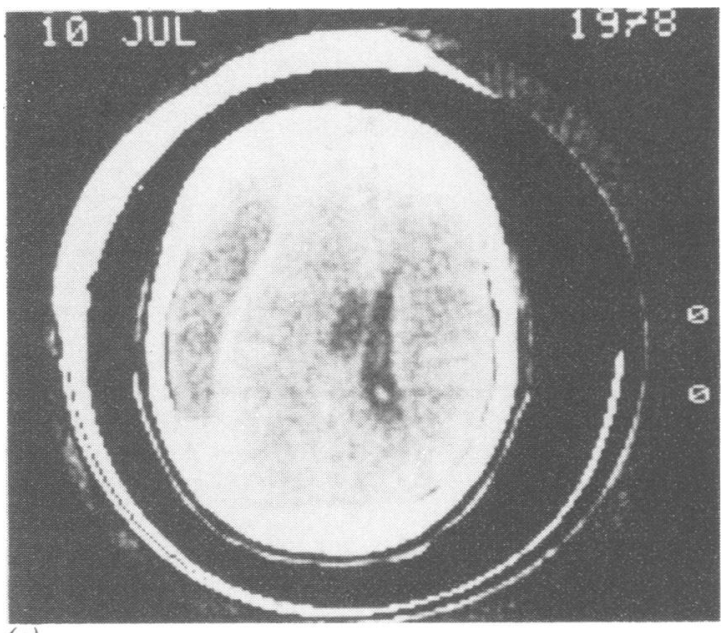

(a)
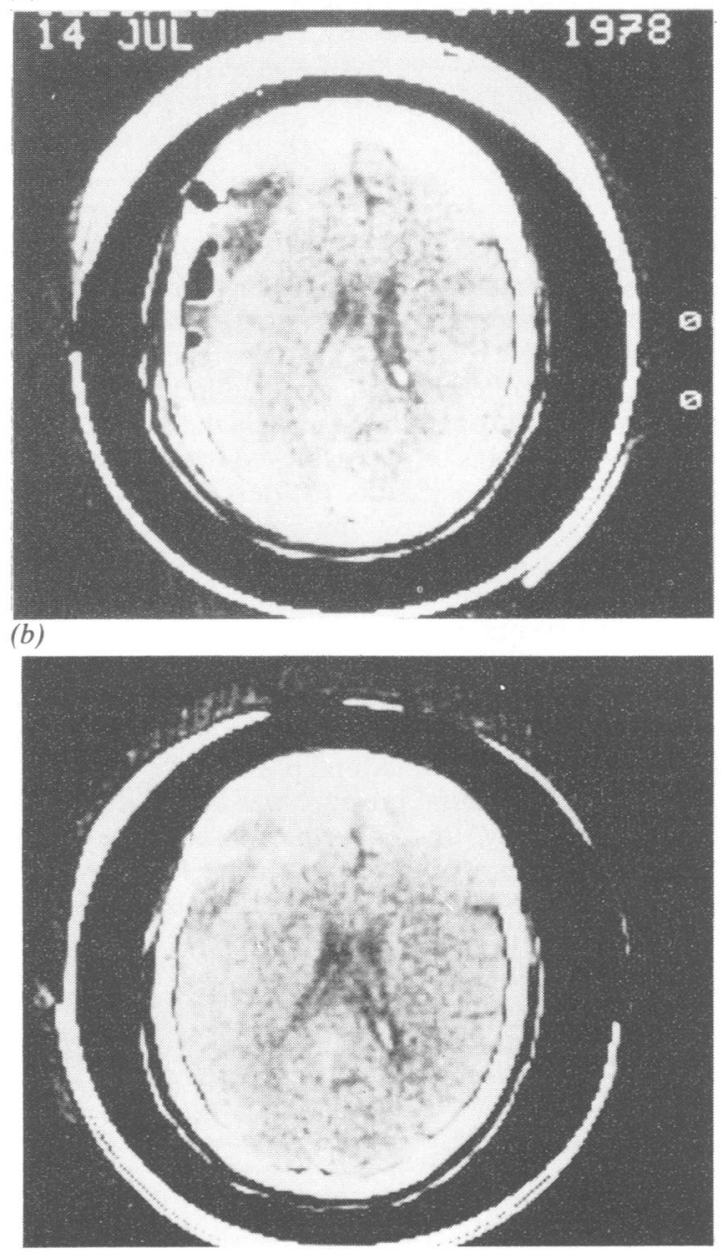

(c) 


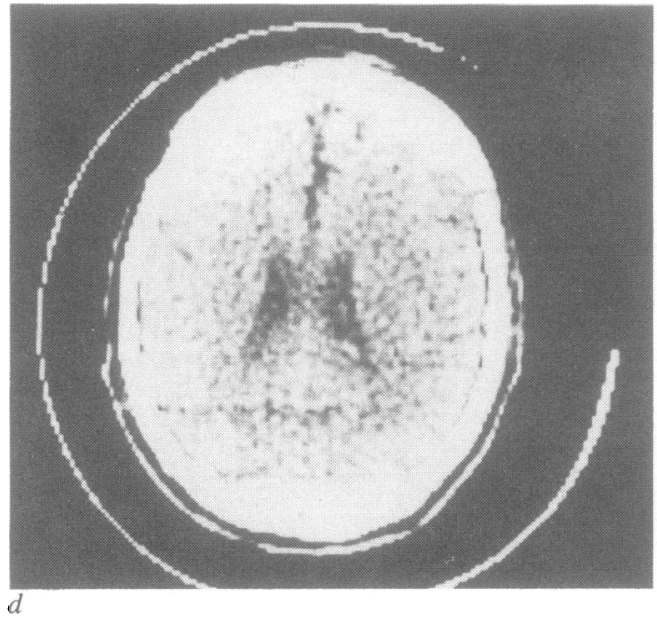

Fig (a) pre-operative appearance of CT scan,

(b) Four days post-operatively, same patient as in (a)

(c) At five weeks, same patient as in (a)

(d) At twelve weeks, same patient as in (a).

\section{Discussion}

From the present study the following points emerge: (1) The outcome of burr hole evacuation of chronic subdural haematoma was good and 23 of our patients made a complete recovery, (2) an appreciable amount of subdural recollection with midline shift was evident at one week post-operatively, even in those patients in whom brain expansion was achieved by per operative intra-thecal saline injection, (3) despite the CT scan appearance at one week, the patients continued to make an uneventful recovery, (4) the collection and the midline shift seen on CT scan resolved without further intervention.

Tabaddor and Shulman ${ }^{9}$ found that clinical improvement occurred after $20 \%$ of the haematoma collection had been drained-an amount often sufficient to lower the subdural pressure close to zero. Although the subdural pressure was not measured in the present study, this important observation might explain the disparity between the clinical picture and the post-operative CT scan appearance in our series. The present study has shown that fluid collections recur in the subdural space after total removal of subdural haematoma, but are not associated with clinical deterioration. Furthermore, the midline dislocation takes time to resolve and is not necessarily related to clinical status. One might argue that this post-operative subdural recollection is merely saline left from irrigation in the subdural cavity. However, analysis of the post-operative subdural fluid obtained from previous cases shows that it cannot be saline; in particular there is often a high protein content. Two studies have reported spontaneous or partial resorption of subdural collection of fluid 56 thus suggesting a mechanism which explains why the CT scan appearances return to normal in all patients.

The present study confirms that the treatment of chronic subdural haematoma by evacuation through one or two burr holes is adequate. Subsequent re-collection of fluid as shown by CT scan often occurs, but should not prompt further intervention unless the patient deteriorates, since it can be expected to resolve spontaneously.

We are grateful to Mr RAC Jones, FRCS, for allowing us to study patients under his care.

\section{References}

${ }^{1}$ Pudenz RD, Shelden $\mathrm{CH}$. The Lucite Calvarium-a method for direct observation of the brain. J Neurosurg 1946;3:487-505.

${ }^{2}$ Rabe EF, Flynn RE, Dodge PR. A study of subdural effusion in an infant with particular reference to the mechanism of their persistance. Neurology (Minneap) 1962;12:79-92.

${ }^{3}$ Apfelbaum RI, Guthkelch AN, Shulman K. Experimental production of subdural haematomas. J Neurosurg 1974; 40:336-46.

${ }^{4}$ Putman TJ, Cushing H. Chronic Subdural Haematoma; its pathology, its relation to pachymeningitis haemorrhagica and its surgical treatment. Arch Surg 1925; 11:329-93.

${ }^{5}$ Bender MB. Recovery from subdural haematoma without surgery. J Mount Sinai Hospital 1960;27:52-8.

${ }^{6}$ Gannun WE, Cook AW, Browder EJ. Resolving subdural collections. J Neurosurg 1962;19:865-9.

${ }^{7}$ Robinson RG. The treatment of sub-acute and chronic subdural haematomas. $\mathrm{Br}$ Med J 1955;1:21-22.

${ }^{8}$ Svien HJ, Gelety JE. On the surgical management of encapsulated subdural haematoma. A comparison of the results of membranectomy and simple evacuation. $J$ Neurosurg 1964;21:172-7.

9 Tabaddor K, Shulman K. Definitive treatment of chronic subdural haematoma by twist-drill craniostomy and closed system drainage. $J$ Neurosurg 1977;46:220-6.

10 Bender MB, Christoff N. Non-surgical treatment of subdural haematoma. Arch Neurol 1974;31:73-9. 